\title{
Effect of magnetic and electric fields on optical properties of semiconductor spherical layer
}

\author{
V.A. Holovatsky, I.B. Bernik \\ Chernivtsi National University, \\ 2, Kotsiubynsky str., 58012 Chernivtsi; \\ Phone: +38(037)2244816, e-mail: ktf@chnu.edu.ua
}

\begin{abstract}
Theoretical investigation of the influence of magnetic and electric fields on the energy spectrum and wave functions of electron in semiconductor spherical layer has been performed. The case of co-directed electric and magnetic fields has been considered. The Schrödinger equation has been solved using the method of expansion for the wave function of electron in the spherical layer under external fields by applying the complete set of wave functions of a quasi-particle in a spherical nanostructure without the external fields. It has been shown that electric and magnetic fields take off the spectrum degeneration with respect to the magnetic quantum number. The external fields rebuild the energy spectrum and deform wave functions of electron. Moreover, their influence on the spherically symmetric state is the largest one. Increasing the magnetic field induction entails a monotonous dependence of the electron energy for the states with $m \geq 0$ and non-monotonous one for the states with $m<0$. The ground state of electron is successively formed by the states with $m=0,-1,-2, \ldots$ with increasing the induction of magnetic field. The enhancement of the electric field mainly diminishes the electron energy. The influence of field on the energy and intensities of the $1 \mathrm{p}-1 \mathrm{~s}$ intraband transition has been studied. It has been shown that there exists a certain value of the electric field, at which the energy of quantum transition doesn't depend on the magnetic field induction.
\end{abstract}

Keywords: spherical layer, intraband transitions, electric field, magnetic field.

Manuscript received 29.10.13; revised version received 02.12.13; accepted for publication 20.03.14; published online 31.03.14.

\section{Introduction}

Semiconductor nanostructures have attracted a particular attention of scientists for about two decades. Recent advances in semiconductor technology allow growing more complex structures than the simple quantum wells, wires or dots. Among them: multiple quantum rings [1], complex quantum wires [2] and multilayered quantum dots [3-5]. The latter are grown using the chemical colloidal method on the basis of $\mathrm{CdS}, \mathrm{CdSe}, \mathrm{ZnS}, \mathrm{ZnSe}$, $\mathrm{HgS}$ and other semiconductor materials and are promising for many technological applications such as infrared photodetectors [6], lasers [7,8], biolabels and biosensors $[9,10]$, etc.

Multilayered spherical quantum dots awake a particular interest of researchers because these structures can be implemented for production of light emitting diodes as they give high-efficient luminescence [11-13]. The simplest multilayered spherical structures are quantum dots composed of the semiconductor core and shell embedded into the matrix [14-16]. If the core serves as a potential barrier and the shell - potential well, the system is called a spherical layer.

External electrical and magnetic fields influence on localization of quasi-particles in quantum wells and affect optical properties of the structures. When the external fields are present, the spherical symmetry is violated and, therefore, calculation of the energy spectrum becomes complicated due to the fitting conditions at the interfaces. The influence of electric field on properties of nanosystems is described by the linear term with respect to the electric field in the 
Schrödinger equation for electron, so the problem can be solved using the variational method or perturbation theory for a simple spherical quantum dot $[17,18]$ and spherical layer [19, 20]. In the article [17], the Stark shift is calculated using the variational method within the framework of the infinite confining potential well model for electron in the spherical quantum dot. The authors of [18] investigated intersubband absorption spectra in the simple spherical quantum dot with definite walls with or without hydrogen on-center impurity. The article [19] is dedicated to the study of the electron energy shift in the spherical layer with impenetrable walls within the model of the perturbation theory. The analytical calculation of the influence of strong electric field on electron states was performed in [20]. But the proposed theory is applicable only for limit case of a "thin enough" layer laying fairly far from the center of the system, which not always corresponds to real nanosystems. The interband transitions in the spherical layer driven by electric field are investigated in $[21,22]$ using the perturbation theory approach.

The study of the magnetic field effect on the electron spectrum and wave functions is much more complicated, as the Hamiltonian of electron comprises not only a linear term, but the quadratic one of the magnetic field induction. Therefore, in the majority of theoretical studies, these investigations are performed for simple quantum dots $[23,24]$. The analogous problem for the spherical layer is solved only using the numerical calculations [25]. The proposed studies of spherical layer don't give any information about optical properties of the structure. Therefore, this problem of the influence of electric and magnetic fields on spherical layer properties needs more detailed investigation.

In this work, we investigate the effect of external electric and magnetic fields on the electron energy spectrum and its probability density of localization in the spherical layer (SL) with infinite confining potentials. Also, the intensities of intraband $1 p-1$ s quantum transitions as functions of the electric field and magnetic field induction are obtained.

\section{Schrödinger equation for electron in spherical layer driven by magnetic and electric fields}

We consider a spherical nanostructure consisting of a core-barrier $\left(r<r_{1}\right)$, a shell-well $\left(r_{1}<r<r_{2}\right)$ embedded into semiconductor matrix $\left(r>r_{2}\right)$, the so-called spherical layer (SL). The directions of the magnetic field induction and electric field are chosen along the $O z$ axis. To obtain the energies and wave functions of electron in SL driven by external fields, the Schrödinger equation with the Hamiltonian (1) is to be solved

$$
\begin{aligned}
& H=\left(\vec{p}-\frac{e}{c} \vec{A}\right) \frac{1}{2 \mu(r)}\left(\vec{p}-\frac{e}{c} \vec{A}\right)- \\
& -e F\left(C r+\frac{D}{r^{2}}\right) \cos \theta+V^{p}(r)+U(r),
\end{aligned}
$$

where $\vec{A}$ is the vector potential, $F$ is the magnitude of the electric field, $V^{p}(r)$ is the self-polarization potential with account of the influence of induced charges on the interfaces, $U(r)$ is the confining potential and

$$
\begin{aligned}
& \mu(r)=\left\{\begin{array}{ll}
m_{0}, & r \leq r_{0}, r_{1}<r \leq r_{2} \\
m_{1}, & r_{0}<r \leq r_{1}, r>r_{2}
\end{array},\right. \\
& \varepsilon(r)=\left\{\begin{array}{l}
\varepsilon_{0}, \quad r_{1} \leq r \leq r_{2} \\
\varepsilon_{1}, \quad r<r_{1}, \quad r>r_{2}
\end{array}\right. \text {, } \\
& U(r)=\left\{\begin{array}{lc}
0, & r_{1} \leq r \leq r_{2} \\
\infty, & r<r_{1}, r>r_{2}
\end{array} .\right.
\end{aligned}
$$

The potential of the field inside the spherical shell in uniform electrostatic field $E$ has the form:

$$
V^{E}(r)=-e F\left(C r+\frac{D}{r^{2}}\right) \cos \theta .
$$

The coefficients $C$ and $D$ are obtained as the solutions of the Poisson equation [26]:

$$
\begin{aligned}
& C=\frac{D\left(\varepsilon_{1}+2 \varepsilon_{0}\right)}{r_{1}^{3}\left(\varepsilon_{0}-\varepsilon_{1}\right)}, \\
& D=\frac{3 \varepsilon_{1}\left(\varepsilon_{0}-\varepsilon_{1}\right) r_{1}^{3} r_{2}^{3}}{r_{2}^{3}\left(\varepsilon_{1}+2 \varepsilon_{0}\right)\left(\varepsilon_{0}+2 \varepsilon_{1}\right)-2 r_{1}^{3}\left(\varepsilon_{1}-\varepsilon_{0}\right)^{2}} .
\end{aligned}
$$

The potential $V^{p}(r)$ was calculated in [27] in the form:

$$
\begin{aligned}
& V^{p}(r)=\frac{e^{2}}{2 r_{1} \varepsilon_{0}}\left(\frac{\varepsilon_{1}-\varepsilon_{0}}{\varepsilon_{1}+\varepsilon_{0}}\right) \times \\
& \times\left\{\frac{r_{1}^{2}}{r_{1}^{2}-r^{2}}+\left(\frac{r_{1}}{r}\right)^{2} F\left(1, \alpha_{1}, \alpha_{1}+1,\left(\frac{r_{1}}{r}\right)^{2}\right)\right\}+ \\
& +\frac{e^{2}}{2 r_{2} \varepsilon_{0}}\left(\frac{\varepsilon_{0}-\varepsilon_{1}}{\varepsilon_{0}+\varepsilon_{1}}\right)\left\{\frac{r_{2}^{2}}{r_{2}^{2}-r^{2}}+\frac{\varepsilon_{0}}{\varepsilon_{1}} F\left(1, \alpha_{2}, \alpha_{2}+1,\left(\frac{r}{r_{2}}\right)^{2}\right)\right\}+ \\
& +\frac{e^{2}}{2 r \varepsilon_{0}} \sum_{n=0}^{\infty}\left\{\left(\frac{r_{1}}{r}\right)^{2 n+1} \frac{\left(\varepsilon_{1}-\varepsilon_{0}\right) n}{\left(\varepsilon_{1} n-\varepsilon_{0}(n+1)\right)}-\left(\frac{r}{r_{2}}\right)^{2 n+1} \times\right. \\
& \left.\times \frac{\left(\varepsilon_{0}-\varepsilon_{1}\right)(n+1)}{\left(\varepsilon_{0} n-\varepsilon_{1}(n+1)\right)}-2\right\} \frac{1}{Z_{n}}
\end{aligned}
$$

$$
Z_{n}=1+\left(\frac{r_{2}}{r_{1}}\right)^{2 n+1} \frac{1}{n(n+1)} \frac{\left(\varepsilon_{1} n+\varepsilon_{0}(n+1)\right)\left(\varepsilon_{0} n+\varepsilon_{1}(n+1)\right)}{\varepsilon_{1}^{2}-\varepsilon_{0}^{2}}
$$

$\alpha_{1}=\frac{\varepsilon_{0}}{\varepsilon_{1}+\varepsilon_{0}}, \quad \alpha_{2}=\frac{\varepsilon_{1}}{\varepsilon_{0}+\varepsilon_{1}}$ 
It is taken into account that the relationship between $\vec{A}$ and magnetic induction $\vec{B}: \vec{A}=[\vec{r} \times \vec{B}] / 2$, and introduced are the new dimensionless magnitudes: $\mathrm{Ry}^{*}=e^{2} /\left(2 \varepsilon a^{*}\right) \quad-\quad$ effective Rydberg energy, $a^{*}=\hbar^{2} \varepsilon /\left(m_{0} e^{2}\right)-$ effective Bohr radius, and parameters $\eta=\hbar B e /\left(2 m c \mathrm{Ry}^{*}\right), \xi=e F a^{*} / \mathrm{Ry}^{*}$. As a result, the Hamiltonian (1) transforms to the form:

$H=-\Delta+\eta L_{z}+(\eta r \sin \theta)^{2} / 4-$

$-\xi\left(C r+\frac{D}{r^{2}}\right) \cos \theta+U(r)+V^{p}(r)$,

where $L_{z}$ is the $z$ component of the angular momentum operator of electron.

If $B=0, F=0$, the Schrödinger equation with the Hamiltonian (11) has the exact solutions:

$\Phi_{n l m}(r, \theta, \varphi)=R_{n l}(r) Y_{l m}(\theta, \varphi)$,

where $R_{n l}(r)=A_{n l} j_{l}\left(k_{n l} r\right)+B_{n l} n_{l}\left(k_{n l} r\right)$, $B_{n l}=$ $=-A_{n l} j_{l}\left(k_{n l} r_{1}\right) / n_{l}\left(k_{n l} r_{1}\right), \quad j_{l}(z), n_{l}(z) \quad-$ Bessel spherical functions of the first and the second kind, respectively; the values $k_{n l}$ are fixed by the condition $R_{n l}\left(r_{2}\right)=0$, and the coefficients $A_{n l}$ - by the normality condition.

In order to study the electron properties in SL driven by electric and magnetic fields, we are going to use the method of expansion of the quasi-particle wave function by applying the complete set of eigen functions of electron in the spherical nanostructure without the external fields, obtained as the exact solutions of the Schrödinger equation [23]. When the external fields are applied, the spherical symmetry of the problem is lowered to the axial one and the orbital quantum number $l$ becomes inconvenient. The new states characterized by a magnetic quantum number $m$ are represented as a linear combination of the states $\Phi_{n l m}(\vec{r})$ :

$\psi_{j m}(\vec{r})=\sum_{n} \sum_{l} c_{n l} \Phi_{n l m}(\vec{r})$.

Substituting (13) into the Schrödinger equation with the Hamiltonian (11), we obtain the secular equation for the electron energy spectrum:

$\left|H_{n l, n^{\prime} l^{\prime}}-E_{j m} \delta_{n, n^{\prime}} \delta_{l, l^{\prime}}\right|=0$,

where the matrix elements $H_{n l, n^{\prime} l^{\prime}}$ have the form:

$H_{n^{\prime} l^{\prime}, n l}=\left(E_{n l}^{0}+m \eta+I_{n^{\prime} l^{\prime}, n l}^{p}\right) \delta_{n^{\prime}, n} \delta_{l^{\prime}, l}-$

$-\xi\left\{\alpha_{l, m} \delta_{l^{\prime}, l+1}+\beta_{l, m} \delta_{l^{\prime}, l-1}\right\} I_{n^{\prime} l^{\prime}, n l}^{F^{\prime}}+$

$+\frac{1}{4} \eta^{2}\left\{\gamma_{l, m} \delta_{l^{\prime}, l+2}+\chi_{l, m} \delta_{l^{\prime}, l}+\lambda_{l, m} \delta_{l^{\prime}, l-2}\right\} I_{n^{\prime} l^{\prime}, n l}^{B}$,

$I_{n^{\prime} l^{\prime}, n l}^{B}=\int_{r_{1}}^{r_{2}} r^{4} R_{n^{\prime} l^{\prime}}(r) R_{n l}(r) d r$,
$I_{n^{\prime} l^{\prime}, n l}^{F}=\int_{r_{1}}^{r_{2}} r^{2} R_{n^{\prime} l^{\prime}}(r)\left(C r+\frac{D}{r^{2}}\right) R_{n l}(r) d r$,

$I_{n^{\prime} l^{\prime}, n l}^{p}=\int_{r_{1}}^{r_{2}} r^{2} R_{n^{\prime} l^{\prime}}(r) V^{p}(r) R_{n l}(r) d r$,

$\alpha_{l, m}=-\sqrt{\frac{(l+1)^{2}-m^{2}}{(2 l+3)(2 l+1)}}, \beta_{l, m}=-\sqrt{\frac{l^{2}-m^{2}}{4 l^{2}-1}}$,

$\gamma_{l, m}=-\sqrt{\frac{\left[(l+2)^{2}-m^{2}\right]\left[(l+1)^{2}-m^{2}\right]}{(2 l+5)(2 l+3)^{2}(2 l+1)}}$,

$\chi_{l, m}=1-\frac{(l+1)^{2}-m^{2}}{(2 l+1)(2 l+3)}-\frac{l^{2}-m^{2}}{4 l^{2}-1}$,

$\lambda_{l, m}=-\sqrt{\frac{\left[(l-1)^{2}-m^{2}\right]\left(l^{2}-m^{2}\right)}{(2 l+1)(2 l-1)^{2}(2 l-3)}}$.

Calculating the energy spectrum and wave functions of electron in SL driven by electric and magnetic fields, the intensity of $1 \mathrm{p}-1 \mathrm{~s}$ intraband quantum transitions can be obtained as follows:

$I_{i-f} \sim\left(E_{i}-E_{f}\right)\left|\int_{V} \psi_{i}^{*}(\vec{r}) r \cos \theta \psi_{f}(\vec{r}) d V\right|^{2}$.

In this work, the energies and intensities of quantum transition are obtained as functions of the magnetic field induction and electric field.

\section{Analysis of the results}

The computer calculations were performed using the physical parameters: electron effective mass $m_{0}=0.13 m_{e}\left(m_{e}-\right.$ the mass of pure electron $)$, dielectric constant $\varepsilon_{0}=10.6$ of CdSe for shell-well and dielectric constant $\varepsilon_{1}=8.25$ of $\mathrm{ZnS}$ semiconductor material for potential barriers.

Expanding the wave functions (13), we took into account enough quantity of terms, providing the condition that the sum of squares of the expansion coefficients was equal to unity with the accuracy not less than $0.01 \%$.

The dependences of the electron energy spectrum on the magnetic field induction (a) and electric field (b) for the cases of applying one or another external field are presented in Fig. 1. The spherical symmetry of the problem is violated by the applied fields, so only two quantum numbers characterizing the electron states should be used, but for convenience, we use the same quantum numbers characterizing the states of electron in a nanostructure driven by electric and magnetic fields as the ones without the external fields. 

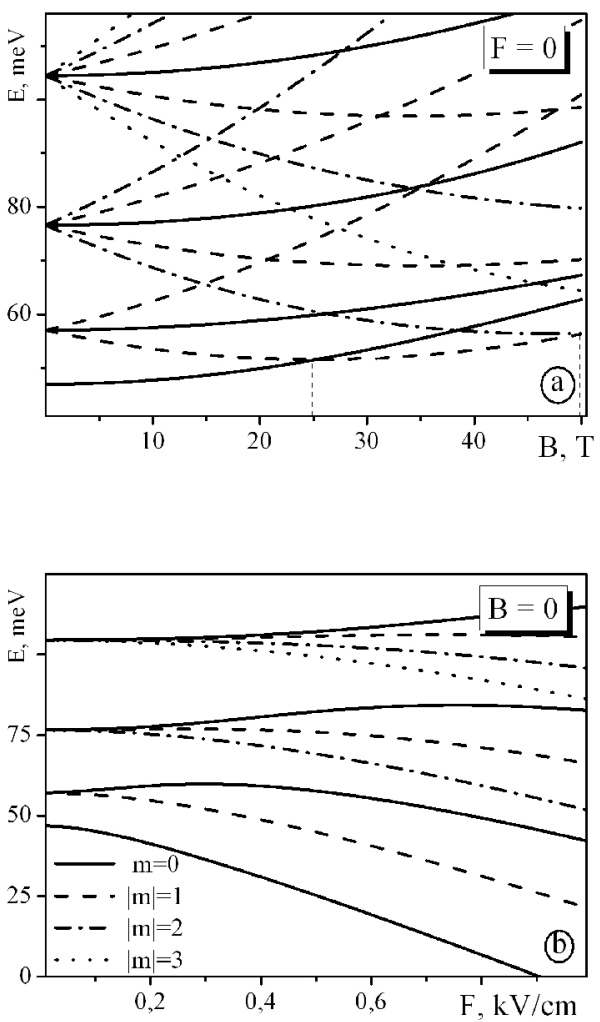

Fig. 1. Energy spectrum of electron in SL with $r_{1}=4 \mathrm{~nm}, r_{2}=$ $12 \mathrm{~nm}$ as a function of the magnetic field induction $(F=0)(a)$, electric field $(B=0)(b)$.

From Fig. 1a, one can see that the energy spectrum of the electron becomes non-degenerate due to the influence of magnetic field. The energies of the states with $m \geq 0$ become higher under the influence of the magnetic field. For the states with $m<0$, the nonmonotonous dependence of the energy on magnetic field is caused by the linear and quadratic terms contributed by the magnetic field in the Hamiltonian (11). When the magnetic field increases, the states with $m=0,-1,-2, \ldots$ successively play the role of the ground state. The ground state of electron with the certain value of the magnetic quantum number transforms into the state with another $m$ when the magnetic field intensity increases at the equal magnitude. Theoretical investigations show that the distance between the points of these transitions increases when the SL radius becomes smaller. Similar behavior of the electron ground state energy was theoretically obtained and experimentally confirmed for the semiconductor quantum rings [28]. Any analogous effects aren't observed for simple quantum dots, for which the ground state is formed by the electron energy state with $m=0$ independently on the magnetic field induction.
Fig. 1b shows that external electrical field partially takes off degeneration of the energy spectrum with respect to the magnetic quantum number, mainly decreasing the electron energy.

Fig. 2 displays the dependences of the electron energy states with $m=0, \pm 1$ on the electric field at the applied external magnetic field with different values of induction. Magnetic field shifts up the energy spectrum. From Fig. 2b, one can see that the ground state is formed by the lowest energy level with $m=-1$ at $B=40 \mathrm{~T}$ in the case of the electric field absence. But the increase of the electric field rebuilds the energy spectrum, to higher extent pulling down the energies of the states with $m=0$. So, at a certain value of $F$, the ground state is formed by the state with $m=0$ as in the case of the external fields absence. In Fig. 2, one can observe the anti-crossing effect for the states with the same value of the magnetic quantum number and the crossing one for the states with different $m$ (the insert of Fig. 2a).

The distributions of probability densities for electron localization in SL in different quantum states are presented in Fig. 3.
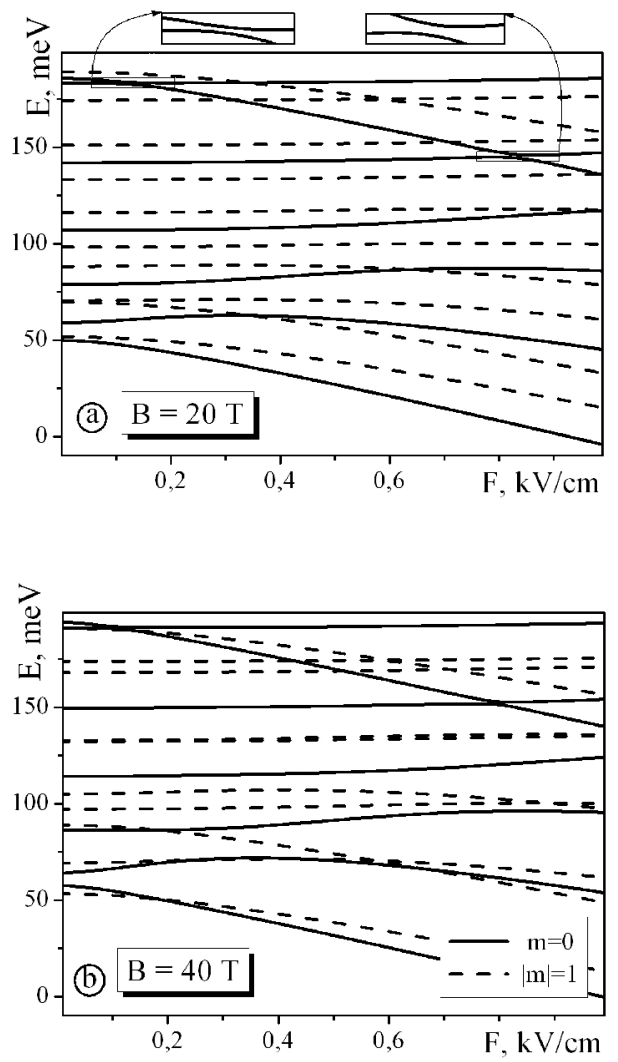

Fig. 2. Energy spectrum of electron in SL with $r_{1}=4 \mathrm{~nm}, r_{2}=$ $12 \mathrm{~nm}$ as a function of the electric field at $B=20 \mathrm{~T}(a), B=$ $40 \mathrm{~T}(b)$. 


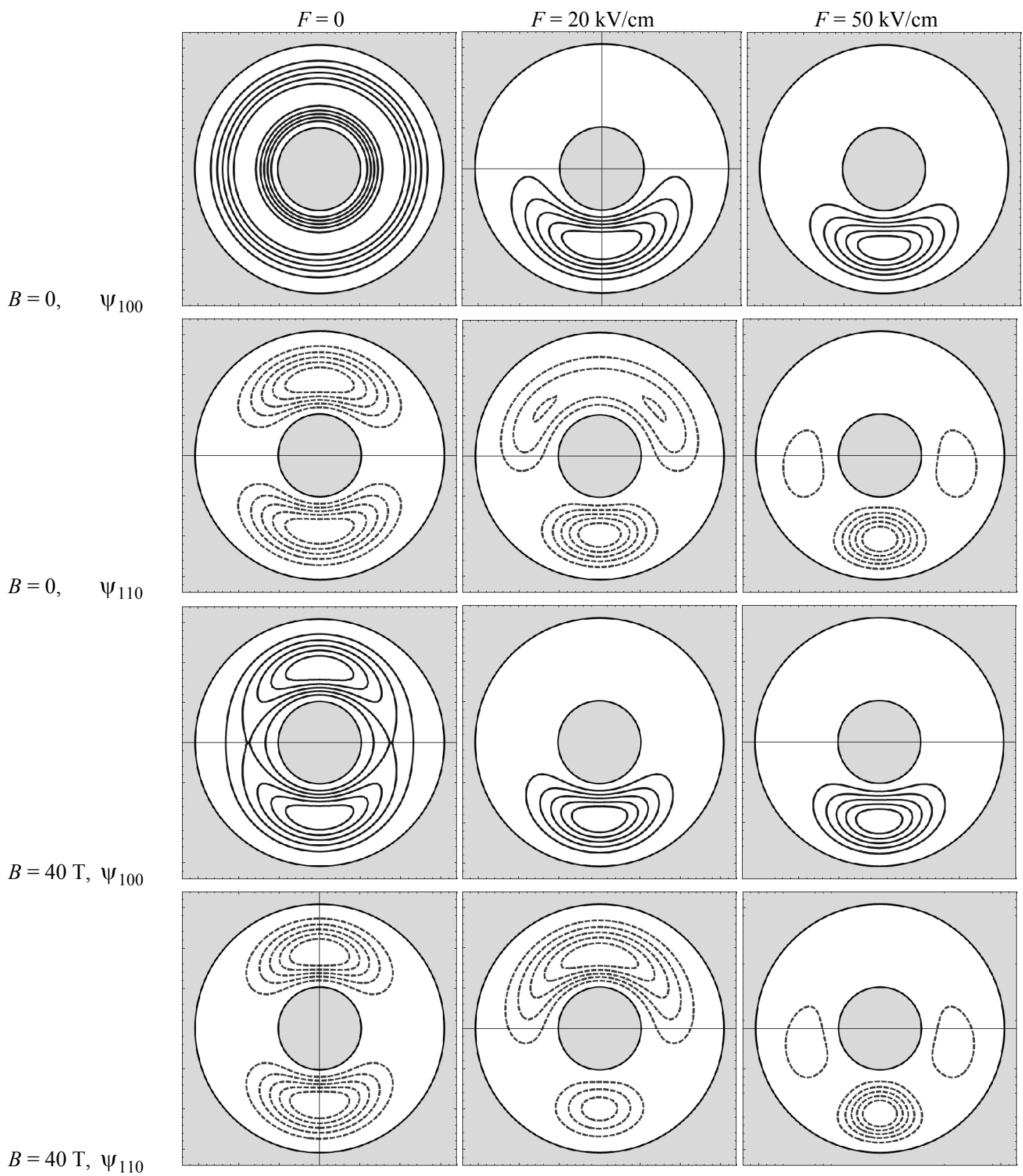

Fig. 3. Distributions of probability densities for electron localization in SL with $r_{1}=4 \mathrm{~nm}, r_{2}=12 \mathrm{~nm}$ for the quantum states with $\psi_{100}, \psi_{110}$ at $B=0,40 \mathrm{~T}$ and $F=0,20,50 \mathrm{kV} / \mathrm{cm}$.

Fig. 3 proves that the electron wave functions are deformed due to the influence of external fields. The turned on electric and magnetic fields deform the distributions of electron densities in SL. In the case of the electric field absence, the angular probability increases near $\theta=0, \pi$ and decreases near $\theta=\pi / 2$, while the magnetic field induction increases. The electron $1 \mathrm{~s}$ state is characterized by the most obvious deformation of its wave function. Its view approaches to the form of the excited $1 \mathrm{p}$ state under the influence of magnetic field.
The energies of the mentioned electron states become closer with enhancement of $B$, too (Fig. 1a). Electric field localizes electron in the potential well made by it near $\theta=\pi$. Magnetic field mainly promotes this effect.

The depicted distributions together with the energies of quantum transitions determine the intensities of intraband transitions. We calculate the intensities of the quantum transitions between the first excited and ground states $(m=0)$. The obtained energies and intensities of the transitions are shown in Fig. 4.

\section{(C) 2014, V. Lashkaryov Institute of Semiconductor Physics, National Academy of Sciences of Ukraine}



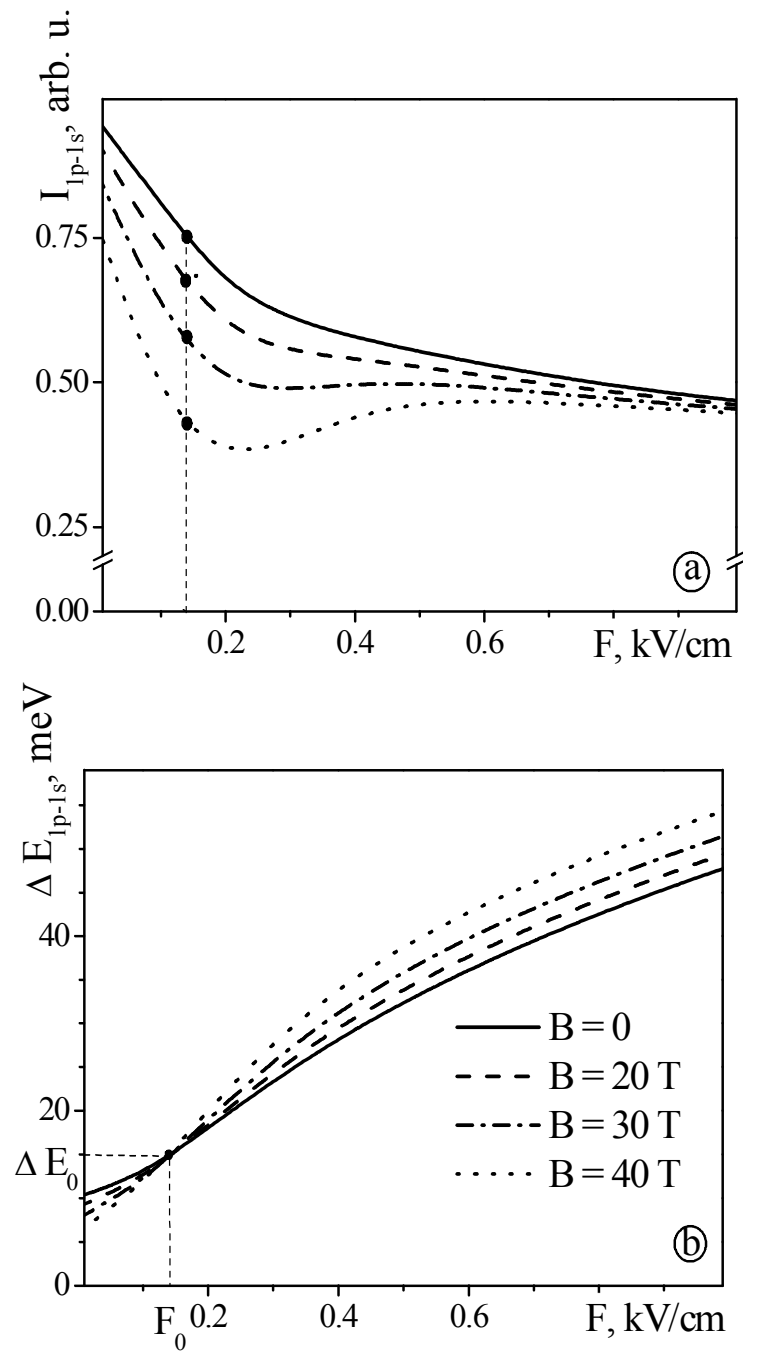

Fig. 4. Dependences of the energies $(a)$ and intensities $(b)$ of the $1 \mathrm{p}-1 \mathrm{~s}$ intraband quantum transition on the electric field at $B=0,20,30,40 \mathrm{~T}$.

From Fig. 4a, one can see that the intensities of the studied transitions decrease with increasing the magnetic field induction, obtaining non-monotonous character of its dependence on the electric field. This behavior is entailed by complicated evolution of energies and distributions of electron probability densities in the ground and first excited states in SL driven by external fields. From Fig. 4b, it is clear that the energy of the $1 p-1 s$ intraband transition increases with electric field strength growth at all the values of the magnetic field induction. At $F_{0} \approx 17 \mathrm{kV} / \mathrm{cm}$, this energy obtains the characteristic value $\Delta E_{0}$ that is constant at arbitrary $B$. The intensity of the quantum transition at $F_{0}$ can be controlled by fitting the magnetic field induction.

\section{Conclusions}

We have studied the electron energy spectrum in semiconductor spherical layer under the influence of electric and magnetic fields. The problem has been solved using the method of electron wave function expansion over the set of eigen functions being the exact solutions of the Schrödinger equation for the same structures without the external fields.

Turning on the magnetic field takes off degeneracy of the energy spectrum with respect to the magnetic quantum number. The electron energies for the states with positive and negative values of the magnetic quantum number differently depend on the magnetic field induction: for the states with $m \geq 0$, the energy monotonously increases, and for $m<0$, the energy decreases at first and then, only when the magnetic field becomes strong enough, enhances. The electric field takes off degeneracy of the spectrum partially. Due to applying the electric field, additional lowering the potential well is introduced, so the energy levels are pulled down.

The distributions of electron probability densities in SL are deformed by both electric and magnetic fields. Electric field localizes electron near $\theta=\pi$, magnetic field with large induction promotes this effect.

The complicated dependence of electron localization on the magnitudes of external fields defines non-monotonous character of the intensities of intraband quantum transitions. The specific value of the electric field exists, at which the intensity of the $1 p-1 \mathrm{~s}$ transition can be changed by applying the magnetic field with different induction at the constant transition energy.

The results of the investigation can be applied for constructing new devices on the basis of multilayered quantum dots. Nevertheless the problem is solved for $\mathrm{CdSe} / \mathrm{ZnS} \mathrm{SL}$, the proposed method can be used for calculating electrical and optical properties of SLs for other materials and sizes. The introduced theory can be applied for further investigation of the features of multilayered quantum dots.

\section{References}

1. T. Mano, T. Kuroda, S. Sanguinetti, T. Ochiai, T. Tateno, J. Kim, T. Noda,. M. Kawabe, K. Sakoda, G. Kido, N. Koguchi, Self-assambly of concentric cuantum double rings // Nano Lett. 5(3), p. 425-428 (2005).

2. M. Heigoldt, J. Arbiol, D. Spirkoska et al., Long range epitaxial growth of prismatic heterostructures on the facets of catalyst-free GaAs nanowires // $\mathrm{J}$. Mater. Chem. 19(7), p. 840-848 (2009).

3. D. Battaglia, B. Blackman, X. Peng, Coupled and decoupled dual quantum systems in one semiconductor nanocrystal // J. Am. Chem. Soc. 127(31), p. 10889-10897 (2005).

4. M. Braun, C. Burda, M. El-Sayed, Variation of the thickness and number of wells in $\mathrm{CdS} / \mathrm{HgS} / \mathrm{CdS}$ quantum dot quantum well system // J. Phys. Chem. A, 105(23), p. 5548-5551 (2001). 
5. M. Uehara, H. Nakamura, H. Maeda, Preparation of $\mathrm{ZnS} / \mathrm{CdSe} / \mathrm{ZnS}$ quantum dot quantum well by using a microfluidic reactor // J Nanosci. Nanotechnol. 9(1), p. 577-83 (2009).

6. S. Krishna, Quantum dot-in-a-well infrared photodetectors // Infrared Phys. \& Technol. 47, p. 151-163 (2005).

7. S. Ivanov, J. Nanda, A. Piryanitski, M. Achermann, L. Balet, I. Bezel, P. Anikeeva, S. Tretiak, V. Klimov, Light amplification using inverted coreshell nanocrystals: towards lasing in the singleexciton regime // J. Phys. Chem. B, 108(30), p. 10625-16030 (2004).

8. Y. Chan, J. Steckel, P. Snee, J-M. Caruge, J. Hodgkiss, D. Nocera, M. Bawendi, Blue semiconductor nanocrystal laser // Appl. Phys. Lett. 86(7), 073102 (2005).

9. U. Resch-Genger, M. Grabolle, S. CavaliereJaricot, R. Nitschke, T. Nann, Quantum dots versus organic dyes as fluorescent label // Nature Methods, 5(9), p. 763-775 (2008).

10. K. Sapsford, T. Pons, I. Medintz, H. Mattoussi, Biosensing with luminescent semiconductor quantum dots // Sensors, 6(8), p. 925-953 (2006).

11. S. Sapra, S. Mayilo, T. Klar, A. Rogach, J. Feldmann, Bright white-light emission from semiconductor nanocrystals: by chance and by design // Adv. Mater. 19(4), p. 569-572 (2007).

12. S. Nizamoglu, E. Mutlugun, T. Ozel, H. Demir, S. Sapra, N. Gaponik, A. Eychmuller, Dual-color emitting quantum-dot-quantum-well CdSe/ZnS heteronanocrystals hybridized on $\mathrm{InGaN} / \mathrm{GaN}$ light emitting diodes for high-quality white light generation // Appl. Phys. Lett. 92(11), 113110 (2008).

13. S. Kim, T. Kim, M. Kang, S. Kwak, T. Yoo, L. Park, I. Yang, S. Hwang, J. Lee, S. Kim, S.-W. Kim, Highly luminescent InP/GaP/ZnS nanocrystals and their application to white light-emitting diodes // J. Am. Chem. Soc. 134(8), p. 3804-3809 (2012).

14. B. Dabbousi, J. Rodrigues-Viejo, F. Micules, J. Heine, H. Mattousi, R. Ober, K. Jensen, M. Bawendi, (CdSe)ZnS Core-shell quantum dots: synthesis and characterization of a size series of highly luminescent nanocrystallites // J. Chem. Phys. B, 101(46), p. 9463-9475 (1997).

15. R. Little, M. El-Sayed, G. Bryant, S. Burke, Formation of quantum-dot quantum-well heteronanostructures with large lattice mismatch: $\mathrm{ZnS} / \mathrm{CdS} / \mathrm{ZnS} / /$ J. Chem. Phys. 114(4), p. 18131822 (2001).
16. G. Murugadoss, Luminescense properties of multilayer coated single structure $\mathrm{ZnS} / \mathrm{CdS} / \mathrm{ZnS}$ nanocomposites // Spectrochimia Acta, Part A, 93, p. 53-57 (2012).

17. G. Vazquez, M. Castillo-Mussot, C. Mendoza, H. Spector, Spherical quantum dot under the electric field // Phys. Status Solidi (c), 1(S1), p. S54-S57 (2004).

18. R. Kostic, D. Stojanovic, Electric field effect on the nonlinear and linear intersubband absorption spectra in CdTe/ZnTe spherical quantum dot // $J$. Nanophotonics, 6(1), 061606 (2012).

19. M. Zoheir, A. Manaselyan, H. Sarkisyan, Electronic states and the Stark shift in narrow band InSb quantum spherical layer // Phys. E, 40(9), p. 2945-2949 (2008).

20. V. Harutyunyan, Nanospherical heterolayer in strong electrostatic field // Appl. Nanosci. 2, p. 399344 (2012).

21. E. Kazaryan, A. Kostanyan, H. Sarkisyan, Interband transitions in a spherical quantum layer in the presence of an electric field: spherical rotator model // Proc. Nat. Academy of Sci. of Armenia, Phys. 42(4), p. 218-226 (2007).

22. M. Zuhair, A. Manaselyan, H. Sarkisyan, Interband transitions in narrow gap InSb spherical layer quantum dot in the presence of electric field // $\mathrm{J}$. Phys.: Conf. Series, 129, 012017 (2008).

23. S. Wu, L. Wan, Electronic structures in a CdSe spherical quantum dot in a magnetic field: Diagonalization method and variational method // J. Appl. Phys. 111(6), 063711 (2012).

24. Z. Xiao, Exciton binding energy in spherical quantum dots in a magnetic field // J. Appl. Phys. 86(8), p. 4509 (1999).

25. J. Planelles, J. Diaz, J. Climente, W. Jaskolski, Semiconductor nanocrystals in a magnetic field // Phys. Rev. B, 65, 245302 (2002).

26. W. Smythe, Static and Dynamic Electricity. McGraw-Hill Book Company, Inc. New YorkToronto-London, p. 140-141, 1950.

27. V. Boichuk, R. Kubay, I. Bilynskii, Influence of the image potential on the energy spectrum of electron in complex spherical microcrystal $\mathrm{CdS} / \beta-\mathrm{HgS} / \mathrm{H}_{2} \mathrm{O}$ // J. Phys. Studies, 3(2), p. 187-191 (1999).

28. A. Lorke, R. Luyken, A. Govorov, J. Kotthaus, J. Garsia, P. Petroff, Spectroscopy of nanoscopic semiconductor rings // Phys. Rev. Lett. 84(10), p. 2223-2226 (2000). 